\title{
Comercio internacional y política económica de la Unión Europea: aportaciones prácticas ${ }^{1}$
}

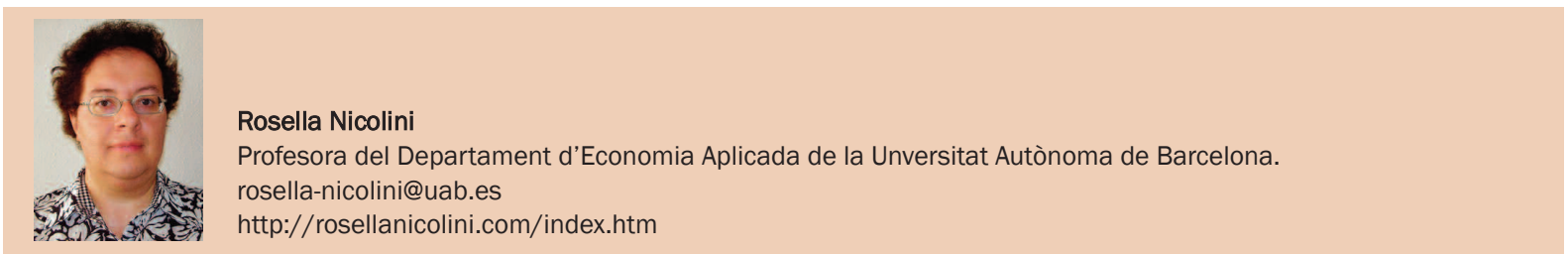

|Fecha presentación: 19/09/2009 | Aceptación: 26/10/2009 |Publicación: 04/12/2009

\begin{abstract}
Resumen
En el actual plan de estudios figuran asignaturas bajo el epígrafe de Comercio internacional en el segundo ciclo, y Economía mundial (internacional) en el primer año del primer ciclo. Existen manuales de referencia que proporcionan un temario muy completo, pero, a veces, con pocos ejemplos y aplicaciones prácticas. Mi propuesta docente es ofrecer un programa en el que se complementen los conceptos teóricos con cuestiones más aplicadas que giren entorno a las políticas económicas referidas a la integración europea. Adicionalmente cabe destacar que las aportaciones son más evidentes e impactantes a la hora de abordar las teorías del comercio internacional bajo condiciones de competencia imperfecta.
\end{abstract}

Palabras claves: nuevas teorías del comercio, competencia imperfecta, integración Unión Europea

\begin{abstract}
Resum
A l'actual pla d'estudis figuren assignatures que s'identifiquen com Comerç internacional en els cursos de segon cicle, i Economia mundial (internacional) en el primer any del primer cicle. Existeixen llibres de referència que proporcionen un temari molt complet, però, a vegades, amb pocs exemples i aplicacions pràctiques. La meva proposta docent és oferir un programa d'assignatures al que es complementin els conceptes teòrics amb temes d'aspecte més aplicat sobre les polítiques econòmiques de la integració europea. Les aportacions són més evidents i impactants a l'hora d'abordar les teories del comerç internacional sota condicions de competència imperfecta.
\end{abstract}

Paraules claus: noves teories del comerç, competència imperfecta, integració Unió Europea

\begin{abstract}
The current course studies include subjects like International trade (advanced level) and Principle of International Economics (usually a first-year course). There are various worldwide textbooks with a very complete table of contents that provide a useful support to teaching but they often lack of examples and empirical applications. My personal teaching innovation is to complement the canonical program of these course programs with notions taken from the economics of the European integration. This strategy revealed to be very useful above all when introducing to students the new trade theories under imperfect competition.
\end{abstract}

Keywords: new trade theories, imperfect competition, EU integration 


\section{1.- Introducción}

En actuales planes de estudios de las titulaciones de Economía y de Ciencias Políticas, la docencia de la Economía internacional aparece bien representada con asignaturas con distinto grado de profundidad y dificultad. De esta manera, se imparten cursos de Introducción a la Economía Internacional (normalmente recogidos bajo el epígrafe de Economía Mundial) así como cursos optativos en los últimos años de la carrera.

El campo objeto de estudio es sin duda muy amplio y cubre aspectos que van desde las teorías clásicas del comercio, a las políticas comerciales, o los temas que relacionan conceptos propios de la Economía internacional con los de otras ramas de la economía, como por ejemplo el desarrollo económico o la organización industrial.

A lo largo del tiempo, los avances de la investigación científica han marcado cada vez más el trato interdisciplinar del estudio de los conceptos básicos. Hablar de ventajas competitivas así como de productividad como fuente de competitividad internacional, no exime de conocer el tipo de estructura productiva que se encuentra debajo de tales condiciones, y de esta manera también parece relevante valorar el papel de las instituciones o la acumulación de factores de producción como son el capital humano o la innovación. Esta complementariedad es de fundamental importancia para interpretar y entender los avances modernos de la teoría del comercio internacional.

La intensa interrelación conceptual que se ha ido elaborando en el estudio de las conexiones entre los conceptos claves como competitividad, ventajas comparativas, comercio y geografía o también comercio y desarrollo, requieren, por parte de los docentes, una doble reflexión sobre la elaboración del contenido de los programas de cada tipo de asignatura y los métodos pedagógicos de enseñanza.

En primer lugar, las asignaturas que tratan temas de Economía internacional se encuentran en distintas titulaciones por lo que hay una clara necesidad de organizar los contenidos de manera que sean asequibles a cada estudiante. En la licenciatura de Ciencias Políticas, por ejemplo, los estudiantes pueden elegir la asignatura de Comercio internacional como optativa, habiendo superado previamente en la mayor parte de los casos un único curso de Introducción a la Economía. Por otro lado, en el caso de la asignatura Economía Mundial, los estudiantes se enfrentan por primera vez al estudio de los temas de Economía internacional. Estos alumnos en no pocas ocasiones carecen de una formación mínima o, se da incluso el caso de que estén cursando de forma simultánea Economía mundial y Principios de Economía. La falta de conocimiento de conceptos o ideas que se adquirirán en cursos superiores (como Microeconomía I y II o Macroeconomía I y II) dificulta la presentación de algunos apartados de la asignatura, sobre todo los relacionados con las nuevas teorías del comercio internacional.

Estas nuevas teorías del comercio internacional han ido consolidándose a partir del final de los años setenta, cuando la evidencia empírica puso en valor la gran importancia del comercio intra-sectorial en la composición de los flujos de intercambio internacionales, algo no interpretable con la teoría ricardiana o el modelo Heckscher- Ohlin (Krugman, 1979, 1980, 1981 y 1991a, así que Brander y Krugman, 1983). Se abre así la incorporación de efectos como las economías de escala o el efecto del aprendizaje en el comercio internacional, desarrollando un enfoque básicamente asentado en las teorías de competencia imperfecta (Fujita, Krugman, y Venables, 1999).
El papel que han ido asumiendo estas últimas teorías en el estudio de los temas de las relaciones comerciales ha favorecido la integración estructural de temas de organización industrial en la conceptualización de las teorías del comercio internacional. La dificultad de poder ilustrar satisfactoriamente estos conceptos, así como desarrollar el marco de análisis de las nuevas teorías del comercio, es el reto más importante para la enseñanza de los cursos de Economía internacional. Esta dificultad no está directamente asociada a la complejidad de los conceptos, sino a la falta de tiempo para poderlos tratar oportunamente en el contexto propio en el que se desarrollan estas asignaturas

¿Y porqué sería importante incluir estas nuevas teorías del comercio internacional en las asignaturas básicas? ¿Se trata verdaderamente conceptos indispensables?

La respuesta no es trivial, y de hecho no es sino hasta las últimas ediciones de los manuales más utilizados (y reseñados en la bibliografía), cuando las nuevas teorías del comercio internacional aparecen formando parte del grueso de la asignatura.

Este nuevo marco conceptual ha ido adquiriendo cada vez más peso por los impresionantes avances de los estudios científicos y por la clara evidencia empírica que sustenta las nuevas teorías del comercio. Además, las nuevas teorías del comercio constituyen un marco de interpretación preferente para valorar los efectos de los procesos de integración económica para los países de la Unión Europea.

La importancia político-social del proceso de integración europea ha convertido el estudio de las causas y los efectos de la integración económica en uno de los temas más destacados de la Economía internacional y cuyos conocimientos son altamente valorados.

Por eso, el propósito de esta nota es ilustrar un posible método que permita incorporar los conceptos de las nuevas teorías del comercio internacional con una atención especial al caso europeo a la vez que proponer un enfoque de análisis adaptado a un público no estrictamente especializado en temas económicos sino con una formación heterogénea.

2.- Complementariedades entre cuestiones relacionadas con la Economía internacional y de integración de la Unión Europea: aspectos a tratar y metodología del aprendizaje

Como ya he anticipado en la introducción de este texto, las últimas ediciones de los manuales más utilizados en los cursos de Economía internacional (Krugman y Obstfeld (2008 y 2006 edición en castellano), Pugel (2009), Tugores (2006), Feenstra y Taylor (2007), Salvatore (2007)) introducen ampliamente los temas recogidos por las nuevas teorías del comercio internacional pero sin poner mucho énfasis en la evidencia empírica, a pesar de que ésta fue en su día el origen de este nuevo enfoque de análisis.

Cabe, de hecho, recordar que el mayor impulso recibido para el estudio de las teorías del comercio bajo un enfoque de competencia imperfecta surgió de los resultados de las estimaciones de los modelos gravitacionales de intercambio bilateral (los así conocidos gravity models) que durante años se presentaron como la solución práctica al problema del estudio de los flujos de intercambio intrasectorial, proporcionando estimaciones robustas aún cuando carecieran de un cuadro de síntesis teórico bien estructurado con el que respaldar estos resultados (Krugman y Obsfeld (2008) o Feenstra y Taylor (2007) ofrecen una descripción muy cuidada de este paisaje). 
La gran novedad introducida por los modelos gravitacionales, que toman este nombre por inspirarse en la ley gravitacional de Newton, es la posibilidad no simplemente de analizar el grado de intercambio intra-sectorial entre países, sino también de medir el valor total de los bienes intercambiados. La ecuación gravitacional del comercio establece que los países con niveles más elevados de producto interior bruto (PIB) o los más próximos físicamente tienen más posibilidad de comerciar entre ellos. Por otra parte, todos los modelos gravitacionales toman la forma de competencia monopolista de la producción en lugar de la competencia perfecta. Esta hipótesis sugiere la siguiente intuición para que se desarrollen acciones de intercambio: los países más grandes (medidos por su PIB) tienen una propensión más elevada para el intercambio porque sus consumidores manifiestan un alto grado de preferencia por consumir muchas variedades de bienes, hecho que se deriva directamente de sus niveles de riqueza más elevados (Feenstra y Taylor, 2007). De esta manera, los países más grandes comercian más porque producen (y exportan) más variedades de productos (efecto de la estructura productiva de competencia monopolista) pero también porque su nivel de demanda (y, con ello, sus importaciones) es elevado. Adicionalmente cabe destacar también que la distancia entre países puede afectar negativamente a la intensidad de intercambio entre ellos, por el hecho de que en estos modelos se supone la existencia de costes de transporte que representan la distancia física, los aranceles u otras barreras, como por ejemplo la lingüística, y generan un aumento del precio final al cual los consumidores pueden acceder a los bienes.

Desde hace unos años, el estudio de los flujos de intercambio intra-sectoriales europeos favorecidos por un pro- gresivo proceso de integración económica (implicando una importante reducción de las barreras al comercio, y, con eso, de los costes de transporte), se ha presentado como uno de los casos de estudio más completo para corroborar la validez de las nuevas teorías del comercio. Desafortunadamente esta dinámica no ha encontrado casi nunca un espacio de relieve en los manuales clásicos adoptados para las asignaturas de Economía internacional, quizás porque estos textos han sido creados principalmente para el mercado de los Estados Unidos y después lanzados mundialmente.

Aún cuando la teoría de la integración económica se ha convertido en un tema fundamental de los estudios de Economía internacional, la experiencia del proceso de integración económica y social fomentado por los países pertenecientes a la Unión Europea representa algo más. Se trata de un tema único porque, de momento, no hay otra experiencia de un proceso de integración entre países que siguen manteniendo su identidad nacional, pero cuyas economías se mueven de manera coordinada y respaldada por una moneda única. Su originalidad así como los aspectos económicos y sociales involucrados en el proceso de integración, constituyen un laboratorio de ideas y casos de estudio que cabe poner en valor ya sea como instrumento y/o como contenido didáctico en los cursos de formación universitarios que abordan temas de Economía internacional.

Las nuevas teorías del comercio internacional, que son las teorías que se asocian más frecuentemente a los temas de integración, son relativamente más complicadas en términos técnicos (Krugman, 1991b) que las teorías tradicionales. Para poderlas entender en profundidad hace falta que un estudiante domine conceptos avanzados de organización industrial (en concreto la idea de competencia imperfecta,

\begin{tabular}{|c|c|}
\hline PROGRAMA & \\
\hline Tema 1. & Introducción: comercio internacional y el debate sobre la globalización. (Cap. 1 [TQ]) \\
\hline Tema 2. & Balanza de pagos e indicadores estadísticos. (Cap. $15[\mathrm{P}])$ \\
\hline Tema 2. & $\begin{array}{l}\text { El modelo ricardiano, la teoría de Heckscher-Ohlin y la teoría de la ventaja comparativa. (Cap. } 3 \\
\text { [P], Cap. } 2[\mathrm{TQ}] \text { ) }\end{array}$ \\
\hline Tema 3 & Los instrumentos de la política comercial. Los acuerdos comerciales. (Cáp 8-11 [P], Cap. 3 [TQ]) \\
\hline Tema 4 . & $\begin{array}{l}\text { Economías de escala, competencia imperfecta y nuevas teorías del comercio } \\
\text { internacional. La teoría de la ventaja competitiva. (Cap. } 5 \text { [P], Cap 10 [BW]) }\end{array}$ \\
\hline Tema 5 . & $\begin{array}{l}\text { Los movimientos internacionales de factores: las migraciones y la inversión directa. Las empresas } \\
\text { multinacionales (Cap. } 14 \text { [P], Cap } 2 \text { [TQ]) }\end{array}$ \\
\hline Tema 6. & Comercio y crecimiento económico. (Cap. 6 [P], Cap 8 [TQ]) \\
\hline Tema 7. & $\begin{array}{l}\text { Comercio internacional, tipos de cambio, tipos de interés y mercado de las divisas. (Cap. 16-20 } \\
\text { [P], Cap. 4 y } 5 \text { [TQ]) }\end{array}$ \\
\hline Tema 8. & La integración económica y distribución de los recursos (Cap. 13 [P], Cap. 6 [TQ]) \\
\hline \multicolumn{2}{|c|}{$\begin{array}{l}\text { Bibliografía básica: } \\
\text { [BW] R. Baldwin and Ch. Wyplosz (2006). Economics of European Integration, 2nd Edition. Mc Graw Hill } \\
\text { [P] Th. Pugel (2004). Economía Internacional. Mc Graw Hill } \\
\text { [TQ] J. Tugores Ques (2006). Economía Internacional. Globalización e } \\
\text { integración regional. Mc Graw Hill }\end{array}$} \\
\hline
\end{tabular}

Figura 1: Síntesis del programa de la asignatura Comercio Internacional en el que expresamente se incluye competencia imperfecta. 
y, en particular de la competencia monopolística) y también debe de estar acostumbrado a manejar instrumentos de calculo matemático de nivel medio-alto. Pre-requisitos que no siempre se cumplen. Lo que deriva en que en manuales como Tugores (2006) o Feenstra y Taylor (2007), se priorice un enfoque muy descriptivo y de discusión (apoyado con datos) para los argumentos presentados.

Seguramente se trata de una estrategia didáctica viable para que los estudiantes se acerquen a este tema, pero que en mi opinión no se adapta completamente a una percepción correcta de la dinámica que conlleva el estudio de las nuevas teorías del comercio internacional.
El mayor valor añadido de las nuevas teorías del comercio internacional se encuentra en la estrecha relación que presentan la distribución de las actividades productivas, la población (en su calidad de trabajadores y consumidores) y las ventajas competitivas que se producen por la formación de aglomeraciones de actividades en lugares específicos y territorialmente limitados (Krugman, 1991b).

Es por eso que los territorios de la Unión Europea, tras el proceso de integración económica, son una de las mejores representaciones del resultado de ese proceso debido a la clara estructura de distribución de actividades productivas en una estructura centro -periferia (Combes y Overman, 2004).

\title{
Curva EE
}

- La curva EE representa la relación entre demanda y distribución de actividad

- La curva EE tiene inclinación positiva: si el Norte concentra una proporción más importante de empresas su mercado será más grande que el mercado en el Sur.

- La curva EE es más inclinada de la linea de $45^{\circ}$; los trabajadores que migran representan
simplemente una parte de la demanda

- La inclinación y posición de la curva EE es totalmente independiente de los costes de transporte.

- En caso de migración de trabajadores hacia el Norte, la curva EE se desplaza a la derecha (EE').

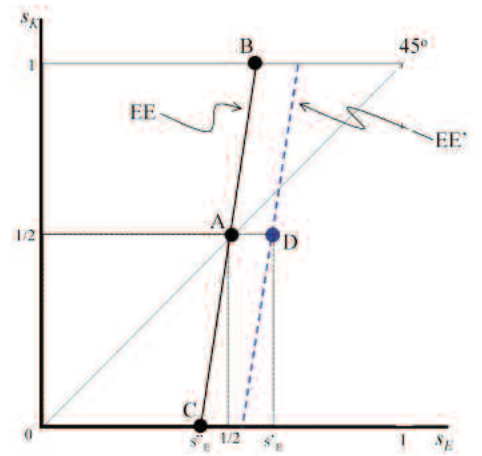

\section{Curva KK}

- La curva KK representa la variación de la distribución de las empresas en relación a la variación de los costes de transporte

Hay un efecto 'tamaño importante' y la curva KK es inclinada positivamente (y más de la diagonal de $45^{\circ}$

Si los costes de transporte suben la curva se acerca a la diagonal

- La distribución de la fuerza trabajo entre las dos regiones no influye en la dinámica de la curva KK

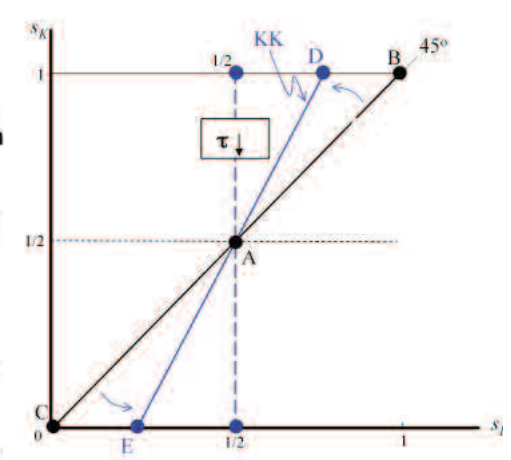

\section{Gráfico EE-KK Diagram}

\author{
- La intersección entre EE \\ y KK determina la \\ distribución de equilibrio \\ $s_{K}$ y $s_{E}$. \\ - Si la economia se \\ encuentra en un otro \\ punto (por ejemplo A) la \\ variación de la demanda y \\ la móvilidad de las \\ empresa la llevaran al \\ punto $B$.
}

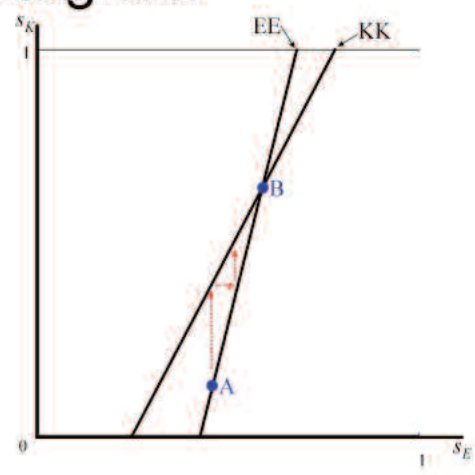

Figura 2: Secuencia introducción creación equilibrio en modelo EE-KK. Fuente: Elaboración propia a partir de Baldwin-Wyplosz (2006). 
Por lo que se refiere a la estructura productiva desigual de los territorios europeos, Baldwin y Wyplosz (2006) introducen en el capitulo 10 un análisis gráfico y empírico con el cual ilustran: (i) el paisaje conceptual desde la teoría de las ventajas comparativas (teorías clásicas del comercio) a las nuevas teorías del comercio, (ii) el tipo de estructura productiva que ha ido consolidándose en Europa gracias al proceso de integración y, (iii) la motivación de la puesta en marcha de las políticas regionales en la Unión Europea para contrarrestar la desigualdad estructural de distribución de recursos.

La principal originalidad introducida en este capítulo es el desarrollo del gráfico identificado como EE-KK (véase Figura 2) con el cual, el estudiante puede de una forma conjunta controlar la dinámica asociada a la integración económica con la variación de la dimensión de las aglomeraciones territoriales de las actividades productivas a medida que se van produciendo modificaciones en los parámetros fundamentales del sistema económico (y variables claves de los modelos de las nuevas teorías del comercio), como costes de transporte, migración (movilidad del factor trabajo) o tamaño de mercado.

La introducción del tema de las nuevas teorías del comercio internacional en el temario elaborado para un curso de Comercio Internacional impartido en la Facultad de Ciencias Políticas, se realiza tal y como queda recogido en el siguiente cuadro (ver Figura 1).

En concreto, siguiendo la estructura del capítulo $10 \mathrm{del}$ libro de Baldwin y Wyplosz (2006), el tema a estudiar se inicia con una presentación de la evidencia empírica sobre la estructura económica de la Unión Europea. Tras esta introducción, se detallan los conceptos de la teoría de la ventaja comparativa (y las causas del comercio internacional bajo este enfoque) y se describe el tránsito hacia la teoría de las aglomeraciones (introduciendo, entonces, el concepto de movilidad de los factores de producción). Llegados a este punto, se considera el estudio de la dinámica de las concentraciones territoriales de actividades como resultado de los cambios sufridos por los distintos factores determinantes (véase costes de transporte o migraciones) para pasar a explicar así la dirección y la intensidad de los flujos de comercio en el área económica europea. La intensidad y la dirección de los flujos comerciales indican que las zonas de origen y destino de los bienes intercambiados coinciden con los lugares donde hay aglomeraciones. El obstáculo técnico, viene por lo tanto en ilustrar cómo y dónde se pueden formar dichas aglomeraciones.

Tal dinámica se ilustra basándose en un enfoque gráfico, utilizando el material que Baldwin y Wyplosz (2006) hacen disponible en la página web dedicada al manual (http://hei.unige.ch/ baldwin/PapersBooks/BW/slides.htm).

En el caso de la Unión Europea, hay evidencia empírica (como ya se ha indicado) que muestra una neta concentración de actividades productivas en el Norte y una menor en el Sur, lo que origina una estructura tipo centro-periferia. El objetivo, ahora, es intentar mostrar porque tal estructura ha originado un incremento importante de los flujos de intercambio entre países una vez ya conseguido el proceso de integración económica. Intuitivamente, los hechos nos indican que, en caso de una reducción de costes de transporte, las empresas eligen donde establecer su centro de activida- des y desde allá servir a todo el mercado.

El problema, técnicamente requiere el estudio de un modelo de competencia imperfecta relativamente complicado, mientras que gráficamente el análisis se simplifica (ver Figura 2) ya que sólo con dos curvas (EE y KK) se puede identificar como varía la distribución de las actividades en un territorio considerando la presencia, por ejemplo, de costes de transporte y migración (parcial de trabajadores).

Es a través de la versión gráfica del modelo que proponen Baldwin y Wyplosz (2006) la forma de hacer accesible este enfoque teórico a cualquier tipo de estudiante que tenga conocimientos económicos equivalentes a los proporcionados por un curso estándar de Introducción a la economía. De esta manera, este enfoque permite ser utilizado con estudiantes de ciencias políticas que siguen el curso de Comercio Internacional o con estudiantes de económicas del primer año en el curso de Economía Mundial.

La idea básica que desarrolla este tipo de enfoque es sencilla: en caso de competencia monopolística desde el lado de la producción, la combinación entre economías de escala y coste de transporte genera condiciones de mercado que favorecen la concentración espacial de las empresas². Entonces, la intensidad de los flujos de intercambio y la dirección de los mismos serán un resultado consecuente de la distribución de las empresas: irán desde donde hay una mayor concentración de empresas hacia donde las empresas son más escasas.

El modelo ilustrado en la Figura 2 (y definido como modelo EE-KK) se basa en los siguientes supuestos:

a) Se consideran dos unidades territoriales (aquí dos regiones) identificadas con Norte y Sur. En cada región se supone que existe el mismo número de trabajadores/consumidores inmóviles. En cada región existe también un sector productivo inmóvil que funciona en competencia perfecta (agricultura, por ejemplo) y un sector industrial que opera en competencia monopolista. Producir en competencia monopolista implica que cada empresa produce una variedad de bienes diferentes sobre el cual tiene la posibilidad de fijar el precio de venta en base al grado de competencia con los demás productores y del grado de sustitución de los bienes en las preferencias de los consumidores. Cada empresa produce utilizando unidades de trabajo y de capital.

b) Los trabajadores/consumidores de ambas las regiones muestran claras preferencias por consumir todos los bienes que se producen en las dos regiones. Existen también costes de transporte que pueden hacer menos accesible la compra de bienes producidos en la región en la cual no son residentes.

c) Las empresas del sector industrial pueden moverse entre las dos regiones en la búsqueda del mercado donde puede alcanzar niveles de ventas más elevados. El movimiento de las empresas influye en el grado de competencia de los mercados (más alta donde se concentran), pero es verdad también que en presencia de una elevada demanda, las empresas pueden explotar las ventajas de las economías de escala. En este contexto se consideran las economías de escala internas, por medio de las cuales se puede reducir el coste medio de producción aumentando la cantidad de bienes producidos.

${ }^{2}$ Los estudiantes trabajaran con los conceptos de economía de escala y competencia monopolista sin entrar en los detalles técnicos. Bastará con que dominen la intuición económica asociada a cada concepto para operar con ellos. 
¿Cómo se puede representar la dinámica de localización de las empresas que quieren explotar las economías de escala en presencia de coste de transporte?

Se puede hacer gráficamente, dibujando dos curvas: la EE y la KK.

1) La curva EE permite identificar la dinámica de localización de las empresas en relación al tamaño de la demanda de mercado, sin considerar los costes de transporte. Para hacerlo se estudia la relación que existe entre la abundancia relativa de empresas en una región, es decir la cuota de empresa en una región con respecto al total de las empresas en las dos regiones. En el Figura 2 , se denota la cuota de empresas en la región Norte con $S_{K}$ en el eje vertical, mientras que la cuota de demanda en la región Norte (respecto a la demanda total) se identifica con $S_{E}$. La acción de las economías de escala produce una relación positiva entre las dos variables lo que se identifica con la pendiente positiva de la curva EE en los gráficos del Figura 2. En caso de que la demanda fuera igualmente repartida entre las dos regiones, también las empresas lo serían, quedando esta situación representada por el punto A del primer gráfico del Figura 2. En el mismo gráfico, el punto B coincide con la situación en la cual todas las empresas se concentran en el Norte y el punto C cuando todas están en el Sur³. La posición de la curva EE en el gráfico varía cada vez ocurre un acontecimiento que puede llevar a un cambio importante en la dimensión de la demanda. Por ejemplo, una aumento de la población en el Norte (por ejemplo, por inmigración) hace que la curva EE se desplace hacia la derecha, identificándose, ahora, con la curva EE'.

2) Para considerar el impacto que tienen los costes de transporte en la decisión de las empresas de trasladar su ubicación, debemos hacer referencia a otra curva, que identificamos con KK. Las empresas aspiran a servir al mercado más grande para poder aprovechar las economías de escala. Pero, por el hecho de comportarse todas de la misma manera, se generan concentraciones de empresas que agudizan el grado de competencia entre ellas. Por eso, los costes de transportes son una limitación a la creación de aglomeraciones, y hacen menos conveniente la movilidad de las empresas ${ }^{4}$. En particular, si los costes de transporte $(\tau)$ fueran prohibitivos, la distribución de las empresas en cada región sería exactamente igual a la cuota de demanda correspondiente en cada región; la curva KK sería entonces igual a la diagonal de $45^{\circ}$ en el gráfico 2 del Figura 2, y, en particular se trataría de la línea entre los puntos C y B. Para valores de coste de transporte nulos, las empresas se repartirían de manera uniforme en las dos regiones: la curva KK sería una línea vertical en $1 / 2$ y pasaría por el punto A. Para cualquier otro valor intermedio de los costes $\tau$ la curva KK se localizará entre estos dos extremos. Entonces, para cualquier valor de $\tau$, si la cuota de demanda en el Norte aumenta, varias empresas del Sur migrarán hacia al Norte hasta llegar a tener los mismos beneficios netos marginales en las dos regiones. Siguiendo con en el Grá- fico 2 de la Figura 2, el punto D corresponde al caso en el cual todas las empresas se concentran en el Norte y el punto E cuando lo hacen en el Sur.

Una vez definida la relación entre la propensión a concentrarse de las empresas, la dimensión de la demanda del mercado y los costes de transporte, se puede identificar el punto de equilibrio, es decir, en el caso del Figura 2, la cuota de empresas que se localiza en el Norte para un nivel de costes fijos (intermedio) y la cuota correspondiente de demanda del Norte. Este punto se obtiene de la intersección de las curvas EE y KK en el tercer gráfico del Figura 2, y, en concreto corresponde con el punto B. Se demuestra, por la dinámica descrita, que este punto es un punto estable a dónde se converge a partir de cualquier otro punto inicial.

En el caso concreto del Figura 2, el punto B nos indica que, en el equilibrio, para un nivel de coste de transporte intermedios, hay una concentración más importante de empresas en el Norte $\left(S_{K}>1 / 2\right)$ así como de demanda de mercado.

Con este enfoque se consigue replicar muy fácilmente la complejidad de los efectos de la integración europea sobre el comercio y la distribución de las actividades. Identificando el efecto de la integración como una reducción de los costes de transporte, esto se traduciría en un movimiento de la curva KK hacia la curva correspondiente vertical dónde $\tau=0$. Intuitivamente, en este caso, el efecto sería un desplazamiento del punto de equilibrio hacia la parte de arriba del gráfico, implicando un efecto de concentración aún más importante en el Norte.

De esta manera, es decir trabajando con ejercicios de estática comparativa, se consigue reproducir gráficamente la interacción entre los factores que determinan la distribución de las actividades en el territorio y así proporcionar un marco de análisis para interpretar la evidencia empírica que se refiere a la situación actual de la Unión Europea.

Asimismo, debido a la sencillez de este enfoque, el estudiante puede realizar de manera totalmente autónoma sencillos ejercicios de estática comparativa, para reflexionar sobre posibles variaciones de distribución de actividades asociadas a la variación de la distribución de recursos.

A título de ejemplo, un ejercicio muy sencillo para facilitar la asimilación de la dinámica del sistema puede ser el siguiente:

Ejercicio: Considera el esquema KK-EE. Identifica cómo varía la estructura productiva de la UE cuando:

- Se reducen los costes de transporte

- Se reducen las fuerzas de aglomeración (por ejemplo, las empresas en el Sur reciben un subsidio para seguir produciendo allí)

- Se refuerzan los flujos de inmigración hacia el Norte.

\section{Conclusiones}

La introducción de argumentos económicos propios del proceso de integración europea en programas didácticos de asignaturas de Economía internacional genera sinergias de gran valor añadido, ya que permite a los estudiantes adquirir nociones de tipo económico en temas de procesos de inte-

\footnotetext{
${ }^{3}$ Hay que recordar también que en el caso de que todas las empresas se ubiquen en una de las dos regiones, sigue existiendo siempre la demanda de bienes por parte de la otra región, para valores de costes de transporte no prohibitivos, puesto que los trabajadores/consumidores no se desplazan.

${ }^{4}$ Con altos costes de transporte, moverse hacia el Norte para una empresa implica perder el mercado del Sur dónde, aunque fuera más pequeño que el del Norte (por ejemplo), podrían beneficiarse de un grado de monopolio más importante.
} 
gración y de tipo técnico en modelos de Economía internacional en condiciones de competencia imperfecta.

Las ventajas docentes asociadas a este tipo de visión del proceso de integración son evidentes sobre todo en el caso de la docencia impartida en los primeros años de carrera en la Licenciatura de Economía o ADE y también en otras licenciaturas dónde la formación de los estudiantes que acceden a esos cursos no les permite disponer de los conocimientos económicos/técnicos suficientes para presentar estos modelos en su formalización canónica cuantitativa.

Además, el ejercicio de fusión de contenido aquí presentado, permite también profundizar en los conocimientos asociados a los procesos de integración europea, sus retos y sus perspectivas, los cuales no siempre están suficientemente desarrollados en los principales textos de Economía internacional generalmente utilizados.

Se deja abierto, como punto de reflexión también, la posibilidad de seguir el camino de la integración de estas dos líneas de temas docentes para poder desarrollar, en el futuro, programas de estudios de Economía internacional con un marco más propio a las características de la Unión Europea y acercar así a los estudiantes a la realidad del tipo de entorno (laboral e institucional) en el cual viven su cotidianidad.

\section{Bibliografía}

Baldwin, Richard; Wyplosz Charles (2006). The economics of European integration. McGraw-Hill Higher Education ( $2^{\text {a }}$ Edition).

Brander, James; Krugman, Paul (1983). A reciprocal dumping model of international trade. Journal of International Economics, 15, pp. 313-321.

Combes, Pierre Ph.; Overman, Henry G. (2004). The spatial distribution of economic activities in the European Union. En J. Vernon Henderson y Jacques-François. Thisse Handbook of Regional and Urban Economics, 4, pp. 2845-2909, Elsevier.

Feenstra, Robert; Alan, Taylor (2007). International Economics. Worth Publisher.

Fujita, Masahisa, Krugman, Paul; Venables, Anthony (1999). The spatial economics. cities, regions and internacional trade. Boston. The MIT Press.

Krugman, Paul (1979). Increasing returns, monopolistic competition, and international trade. Journal of International Economics, vol.9, pp. 469-479.

Krugman, Paul (1980). Scale economies, product differentiation and the pattern of trade. American Economic Review, 70, pp. $950-959$.

Krugman, Paul (1981). Intraindustry specialization and the gains from trade. Journal of Political Economics, 89, pp. 959-973.
Krugman, Paul (1991a). Geography and trade. Boston, The MIT Press.

Krugman, Paul (1991b). Increasing returns and economic geography. Journal of Political Economics. 99 (3), pp. 483-499.

Krugman, Paul;Obstfeld, Maurice (2008). International Economics. Theory and Policy. Boston, Pearson Education ( $8^{\mathrm{a}}$ Edición).

Krugman, Paul; Obstfeld, Maurice (2006). Economia Internacional. Teoría y Politica. Madrid, Pearson Addison Wesley ( $7^{\text {a }}$ Edición).

Pugel, Thomas (2009). International Economics. McGraw Hill (14 ${ }^{\mathrm{a}}$ Edition).

Salvatore, Dominik (2007). International Economics. Wiley (9 $9^{\text {a }}$ Edition).

Tugores Quaes, Juan (2006) Economía Internacional. Globalización e integración regional. Madrid. McGraw-Hill Interamericana de España. 